\title{
Spontaneous in Utero Rupture of Fetal Meningomyelocele Sac: A Case Report.
}

\author{
Randal A. Loy, M.D., Hamid A. Hadi, M.D., Hossam E. Fadel, M.D., Sara A. Martin, R.D.M.S. \\ Augusta, GA
}

\begin{abstract}
Route of delivery in cases of intact fetal meningomyelocele is controversial. Currently, many obstetricians are concerned about rupture of the meningomyelocele during vaginal delivery and elect to perform caesarean section. We present a case of meningomyelocele in which the sac was spontaneously ruptured two days prior to cesarean section, suggesting that in some cases vaginal delivery may not be responsible for rupture of the sac.
\end{abstract}

Meningomyelocele results from failure of closure of the caudal portion of the neural tube. ${ }^{1,2}$ This defect occurs 1-2 per 1000 births in the United States and may be associated with subsequent development of musculoskeletal and neurological handicaps. ${ }^{3} \mathrm{Re}-$ cent advances in technological development of obstetric ultrasound have contributed a great deal in antenatal detection of such anomalies. However, the optimal approach regarding route of delivery of such infants still remains a controversial subject. The potential risks of vaginal delivery in infants with meningomyelocele are claimed to consist of traumatic rupture of the sac and infection. Therefore, caesarean delivery is offered to the patients as a nontraumatic and beneficial procedure. We present a case of intact fetal meningomyelocele which spontaneously ruptured in the absence of uterine contractions prior to caesarean section operation.

\section{Case report}

A 34 year old woman, gravida 2, para 1 was transferred to the Medical College of Georgia's

From the Department of Obstetrics and Gynecology Medical College of Georgia, Augusta, GA 30912

Reprint Requests: Hamid A. Hadi, M.D.

Department of Obstetrics and Gynecology

East Carolina University, School of Medicine

Greenville, NC 27858-4354.
Obstetrics Department for futher management of pregnancy complicated by fetal neural tube defect. At the time of presentation, she was approximately 33 weeks pregnant with a known fetal sacrococcygeal meningomyelocele and mild hydrocephaly. The past medical history was noncontributory, except for primary infertility and previous ovarian wedge resection. There was no known history of neural tube defects in the family.

The initial obstetric ultrasound examination demonstrated a fetal cranium with dilated lateral ventricles. The lateral ventricular width/hemispheric width ratio $(\mathrm{LVW} / \mathrm{HW})$ was $56.1 \%$. (normal = $25-37 \%$.) The cortical mantle was $10 \mathrm{~mm}$. A sacral meningomyelocele with an intact sac was noted which measured $4 \times 4 \mathrm{~cm}$ in both vertical and transverse diameters. (Figure 1)

A repeat ultrasound examination was performed three weeks later which revealed a slightly increased ventriculomegaly (LVW/HW ratio of $59.8 \%$ ). Although spinal disraphism was noted, the previously well-defined meningomyelocele sac was not visualized. (Figure 2) The patient did not have uterine contractions nor had she been in labor. Amniocentesis for fetal pulmonary maturity was performed, and the delivery was effected through a primary, lower-segment, transverse caesarean section. The result was a viable $3320 \mathrm{gm}$ male infant with 8 and 9 apgar scores in 1 and 5 minutes, respectively. Ruptured sacrococcygeal meningomyelocele was noted at delivery. The neonate underwent successful neurosurgical repair of the neural tube defect 


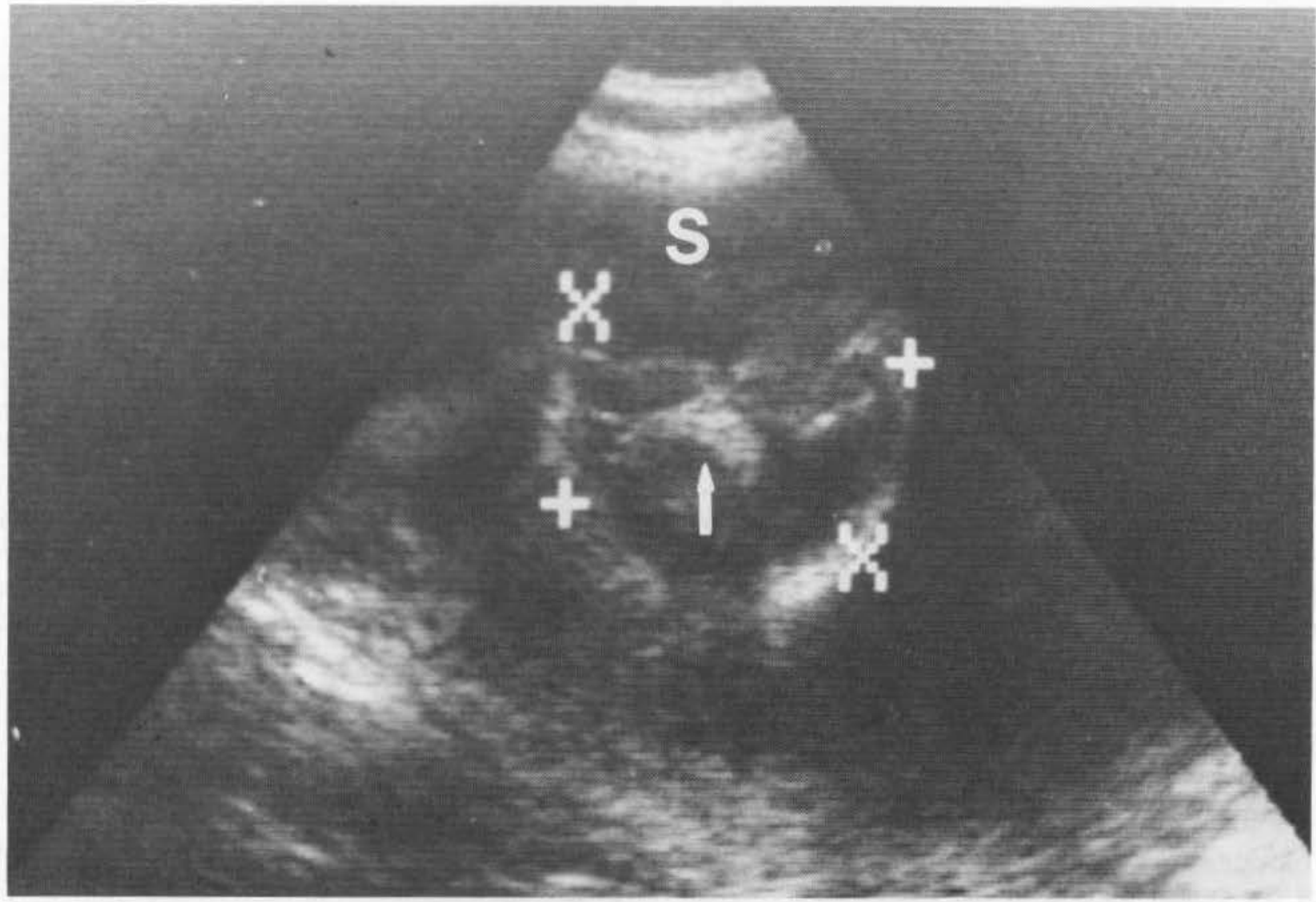

Figure 1. Initial ultrasound examination. Transverse section at the level of the fetal sacrum. The meningomyelocele sac is intact. It measured $4 \times 4 \mathrm{cms}$. $\mathrm{S}=$ body of sacrum. The arrow points to neuronal and meningeal elements in the sac.

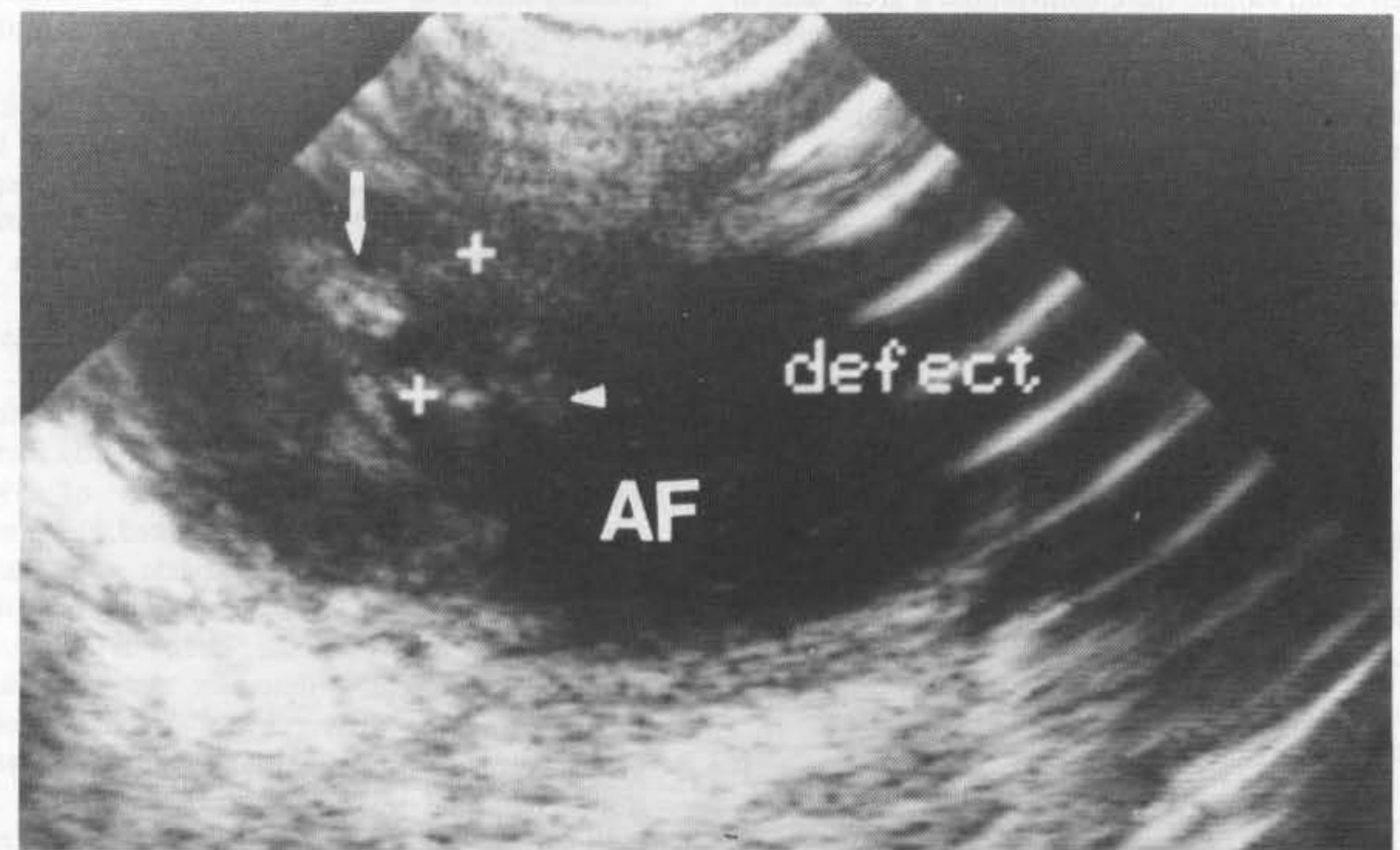

Figure 2. Repeat ultrasound examination ( 3 weeks later). Transverse view at the level of the defect. Meningomyelocele sac has spontaneously ruptured. The characteristic U-shaped defect (arrowhead) is noted. The arrow points to the body of sacral vertebra. AF = amniotic fluid.

Page 82 - JIMA: Volume 22, 1990 
on the first day of life. Placement of a ventriculoperitoneal shunt was not required. The patient was discharged from the hospital five days after the operation in stable condition.

\section{Discussion}

Due to poorly defined landmarks, the fetal sacral spine often cannot be completely evaluated by sonography. ${ }^{*}$ However, with this relatively large defect involving splaying of the lower spine, saccular protrusion from the sacrococcygeal area, and improved ultrasound resolution, the diagnosis of meningomyelocele was secure.

The obstetric management of meningomyelocele and route of delivery are controversial subjects. It is believed that vaginal delivery of the infant with meningomyelocele can be traumatic and may result in rupture of the sac. Therefore, caesarean delivery has been offered as a potentially beneficial procedure. Our case shows that caesarean delivery of such infants does not always guarantee an intact meningomyelocele sac, as it may rupture spontaneously prior to the procedure. Previously, we have reported that a meningomyelocele smaller that $4 \mathrm{~cm}$ in diameter was delivered vaginally without rupture of the sac. ${ }^{6}$ Another concern regarding vaginal delivery of such infants is the risk of infection through the birth canal. This dilemma still remains and further studies are required to confirm this claim.
In summary, to the best of our knowledge, this is the first documented case of spontaneous rupture of a meningomyelocele sac in utero. This study shows that the meningomyelocele sac may spontaneously rupture in utero prior to delivery. If obstetricians decide to select caesarean section as the route of delivery so as to avoid rupture of the sac, it is essential that the ultrasound examination be repeated a short time prior to delivery.

\section{References:}

1. Osaka K, Tanimura T, Hirayama A, Matsumoto, S: Menigomyeolocele before birth. J Neurology 1978;49:711-724.

2. Naggan L, MacMahon B: Ethnic differences in the prevalence of anencephaly and spina bifida in Boston, Massachusettes. N Engl J Med 1967;277:1119-1123.

3. Leonard C O, Freeman J M: Spina bifida: A new disease. Pediatrics 1981;68:136-138.

4. Hobbins JC, Grannum PAT, Berkowitz RL, et al: Ultrasound in the diagnosis of congenital anomalies. AJ Obstet Gynecol 1979;134:331-341.

5. Chervenak F A, Duncan C, Ment L, et al: Perinatal management of meningomyelocele. Obstet Gynecol 1984;63:376.

6. Hadi HA, Loy RA, Long EM, et al; Outcome of fetal meningomyelocele after vaginal delivery. J Reprod Med 1987;32:597. 